\title{
Hepatectomía en dos tiempos para las metástasis hepáticas de origen colorrectal
}

\author{
Santiago Cubas*, Fernando Bonilla†, Luis Ubilloł, \\ Alonzo Rodríguez§, Luis Cazabán", César Canessa**
}

\section{Resumen}

El tratamiento de las metástasis hepáticas de origen colorrectal ha ido cambiando en estos últimos 20 años. En la actualidad la cirugía constituye un pilar fundamental en el tratamiento de estos enfermos. La sobrevida de los pacientes pasibles de una resección completa del tumor puede ascender a $40 \%$ en cinco años. Estos resultados oncológicos tan alentadores han llevado a los equipos quirúrgicos a esforzarse por lograr resecciones hepáticas extremas contraindicadas en tiempos anteriores. Es, en esta línea de trabajo, que se desarrolló la cirugía de remodelación hepática que consiste en alterar el flujo portal buscando una hipertrofia del hígado sano y atrofia del enfermo. El siguiente caso pretende mostrar una de las dos cirugías de remodelación más utilizadas en el mundo, que es la hepatectomía en dos tiempos con oclusión del flujo portal. Se discute su indicación, la oportunidad de las cirugías, el beneficio oncológico y la viabilidad de su realización en nuestro medio.

Palabras clave: Neoplasias colorrectales

Metástasis de la neoplasia

Hepatectomía en dos etapas

Vena porta

Resultado del tratamiento

Key words: $\quad$ Colorectal neoplasms

Neoplasm metastasis

Two-stage hepatectomy

Portal vein

Treatment outcome

\footnotetext{
* Prof. Adjunto de Clínica Quirúrgica "B". Hospital de Clínicas, Montevideo, Uruguay.

† Prof. Adjunto de Clínica Quirúrgica "B". Hospital de Clínicas, Montevideo, Uruguay.

$\ddagger$ Prof. Adjunto de la Cátedra de Oncología de la Universidad de la República, Montevideo, Uruguay.

$\S$ Prof. Adjunto del Departamento de Imagenología del Hospital de Clínicas, Montevideo, Uruguay.

T Prof. Agregado de la Clínica Quirúrgica "B". Hospital de Clínicas, Montevideo, Uruguay.

** Prof. de la Clínica Quirúrgica "B". Hospital de Clínicas. Montevideo, Uruguay.

Clínica Quirúrgica "B". Hospital de Clínicas, Montevideo, Uruguay.

Departamento de Cirugía del Hospital Español. Montevideo, Uruguay.

Los autores declaran no tener conflicto de intereses.

Correspondencia: Dr. Santiago Cubas. Tulipanes 2825, barrio Los Olivos. Lote 29C. CP 12100. Montevideo, Uruguay. Correo electrónico: santicubas@gmail.com
} 


\section{Introducción}

El 50\% de los pacientes con cáncer colorrectal desarrollan metástasis hepáticas en la evolución de su enfermedad y el $25 \%$ de estos las presentan al momento del diagnóstico $^{(1,2)}$. La metastasectomía quirúrgica es la principal herramienta terapéutica en estos enfermos generando un aumento considerable en la sobrevida, que puede ascender a $40 \%$ en cinco años si se logra una resección completa del tumor ${ }^{(3-5)}$.

La mejora en los resultados de morbimortalidad de la cirugía hepática, así como el desarrollo de tratamientos de quimioterapia más efectivos, ha llevado a ampliar el número de pacientes en los que se indica la cirugía de las metástasis. Adicionalmente, el concepto de resecabilidad ha cambiado, siendo fundamental el volumen hepático remanente y no el número, tamaño y ubicación de las metástasis, como se consideraba anteriormente. Si a esto le sumamos la cirugía de remodelación hepática, que consiste en alterar el flujo hepático portal para buscar una hipertrofia de sectores sanos de hígado y atrofia de los enfermos, el número de pacientes con metástasis hepáticas potencialmente resecables aumenta considerablemente ${ }^{(6,7)}$

\section{Caso clínico}

Mujer de 63 años, extabaquista, con un buen performance status (PS 1), a quien se le diagnostica un tumor de recto bajo (adenocarcinoma moderadamente diferenciado) con infiltración del canal anal, en mayo de 2016. En la estadificación inicial presentaba dos metástasis hepáticas sincrónicas en el hígado derecho.

Dado lo sintomático del recto (pujos, dolor y tenesmo) se decidió comenzar el tratamiento por este. Recibió un plan de neoadyuvancia con radio y quimioterapia (50,5 Gy más $3.000 \mathrm{mg} /$ día de capecitabine). Presentó una respuesta parcial al tratamiento y en mayo de 2016 se le realizó una amputación abdominoperineal video asistida, con buena evolución posoperatoria, quedando con una colostomía terminal en fosa ilíaca izquierda.

La anatomía patológica de la pieza mostró un adenocarcinoma moderadamente diferenciado que infiltra la subserosa del órgano sin evadirlo con márgenes libres de tumor en la resección y 0/9 ganglios positivos. La estadificación final de la pieza posoperatoria del recto fue yp T3 N0.

A partir de agosto de 2016 y hasta enero de 2017 recibe quimioterapia posoperatoria con un esquema de folfoxiri (fluoruracilo, oxaliplatino e irinotecán) obteniéndose una estabilización de su enfermedad hepática. En los estudios imagenológicos previos a la cirugía hepática se observan tres metástasis hepáticas. Dos de ellas grandes, que ocupaban casi todo el hígado derecho, y una de $45 \mathrm{~mm}$ en segmento 3 contra la cara izquierda del ligamento falciforme próximo al receso de Rex (figura 1).

De la volumetría hepática resaltaba un volumen hepático total (VHT) de $1.410 \mathrm{~cm}^{3}$ y un volumen hepático residual (VHR) de $300 \mathrm{~cm}^{3}$ con un cociente VHT/VHR de $21 \%$. Con el fin de evitar una falla hepática posoperatoria se decide realizar una cirugía en dos tiempos. En marzo de 2017 se realiza la metastasectomía del segmento tres, colecistectomía y ligadura de la rama derecha de la vena porta. A las ocho semanas de la primera hepatectomía se confirma por tomografía la hipertrofia del hígado izquierdo (figura 2) y se procede a realizar, en mayo de 2017, la hepatectomía derecha (figuras 3 y 4).

La paciente presentó una buena evolución posoperatoria, sin complicaciones mayores, y fue dada de alta a los 12 días de la segunda cirugía hepática (figura 5). Al momento actual, a 17 meses de la segunda cirugía, la paciente se encuentra viva. Presenta una imagen de $3 \mathrm{~cm}$ en el hígado izquierdo, sugestiva de una metástasis metacrónica, y está siendo valorada por el equipo multidisciplinario para una re-resección.
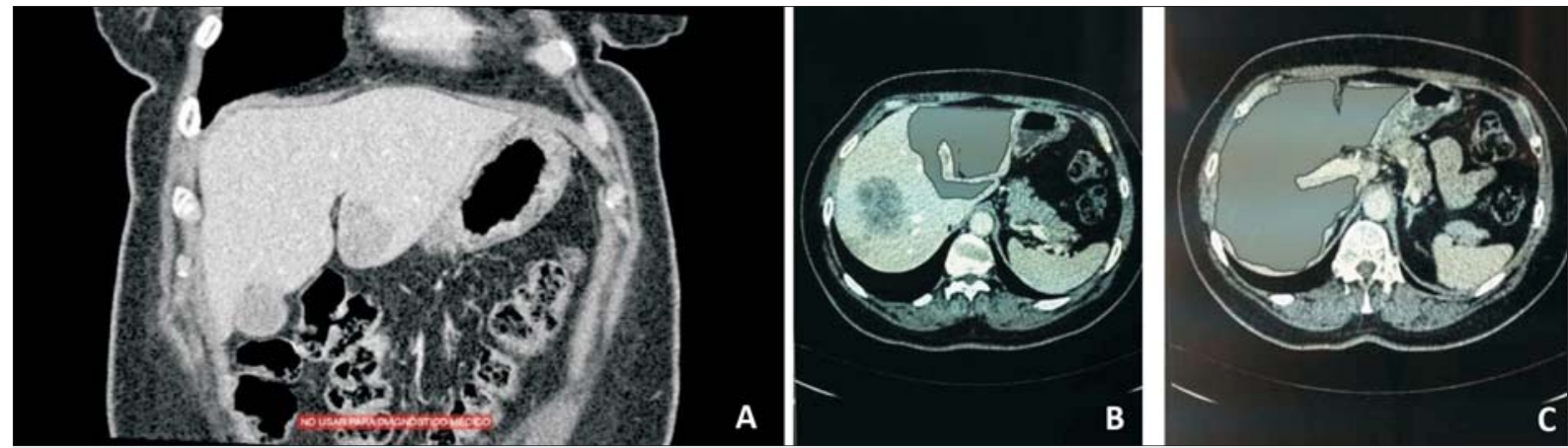

Figura 1. Situación del hígado previo al tratamiento. A) Dos metástasis a la derecha de la vena suprahepática media que ocupan casi todo el hígado derecho. B) Metástasis de $45 \mathrm{~mm}$ sobre la cara izquierda del ligamento falciforme en contacto con el receso de Rex. C) Cálculo del volumen hepático residual mediante volumetría. 

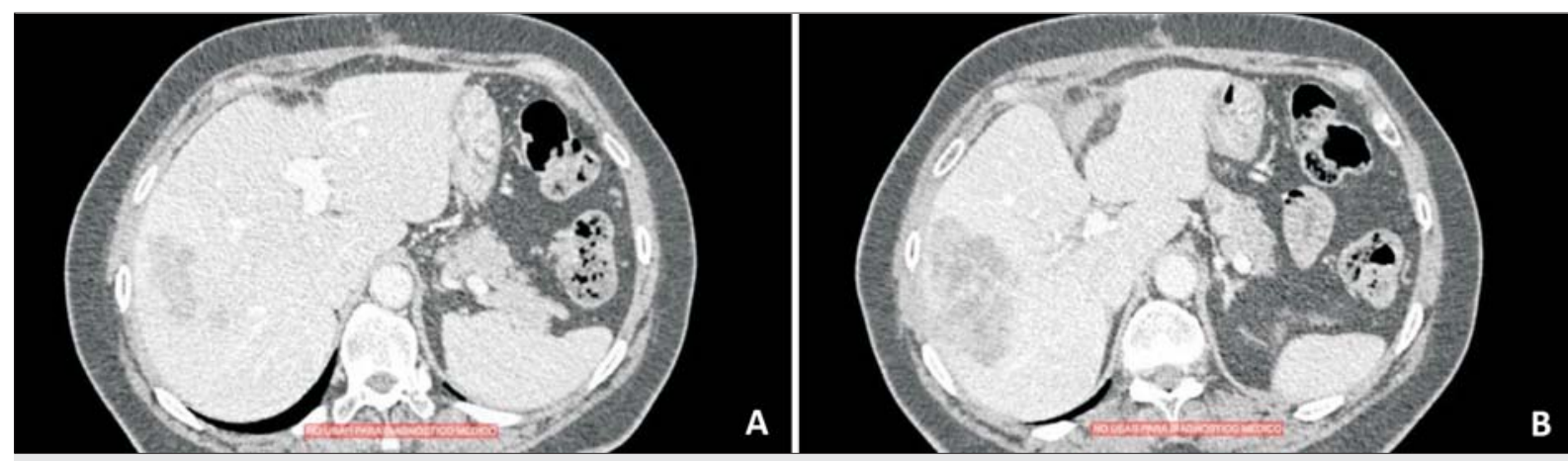

Figura 2. Tomografía computada a las siete semanas de la primera cirugía. A) Se evidencia una hipertrofia del hígado izquierdo (segmentos 2, 3 y 4), así como una densidad tomográfica diferente entre el hígado derecho e izquierdo. B) Se evidencia la cicatriz posoperatoria en la topografía de la metastesectomía (segmento 3).

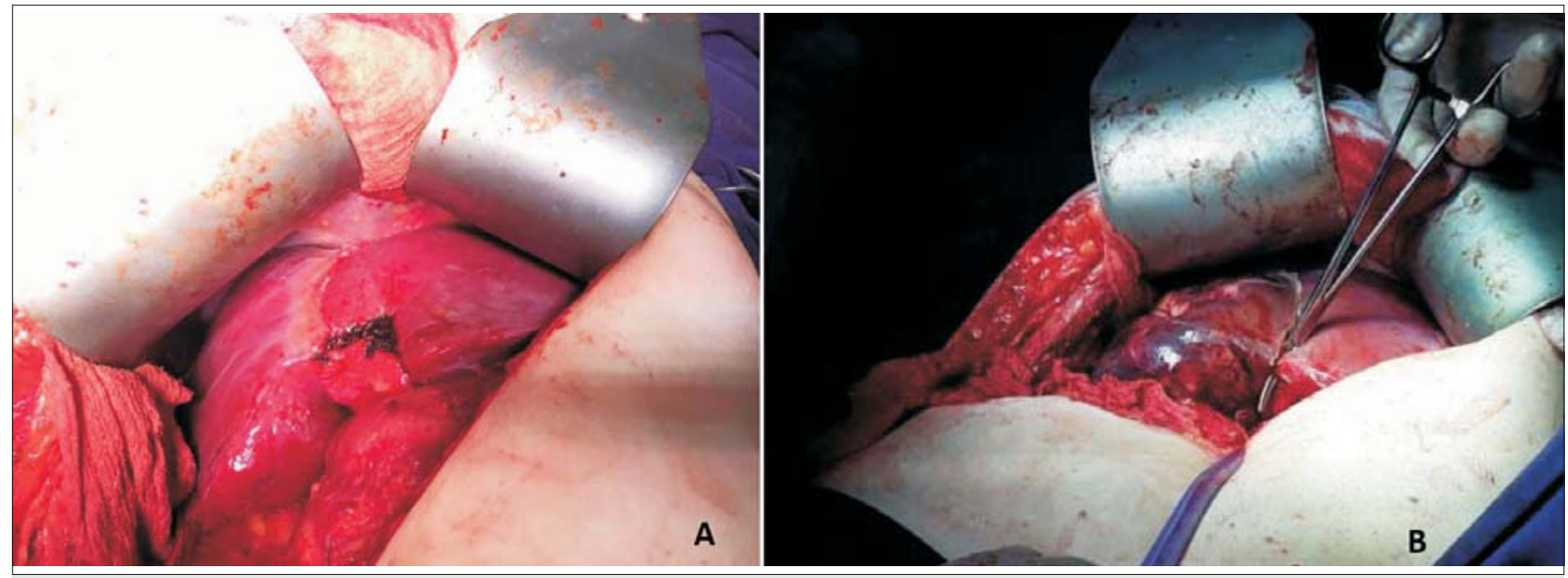

Figura 3. Segunda cirugía de la HDT. A) Se visualiza la cicatriz de la metastasectomía del segmento 3 y la hipertrofia del hígado izquierdo. B) Comienzo de la hepatectomía derecha en la línea de Cantie. Se evidencia la diferencia en la perfusión del hígado derecho.

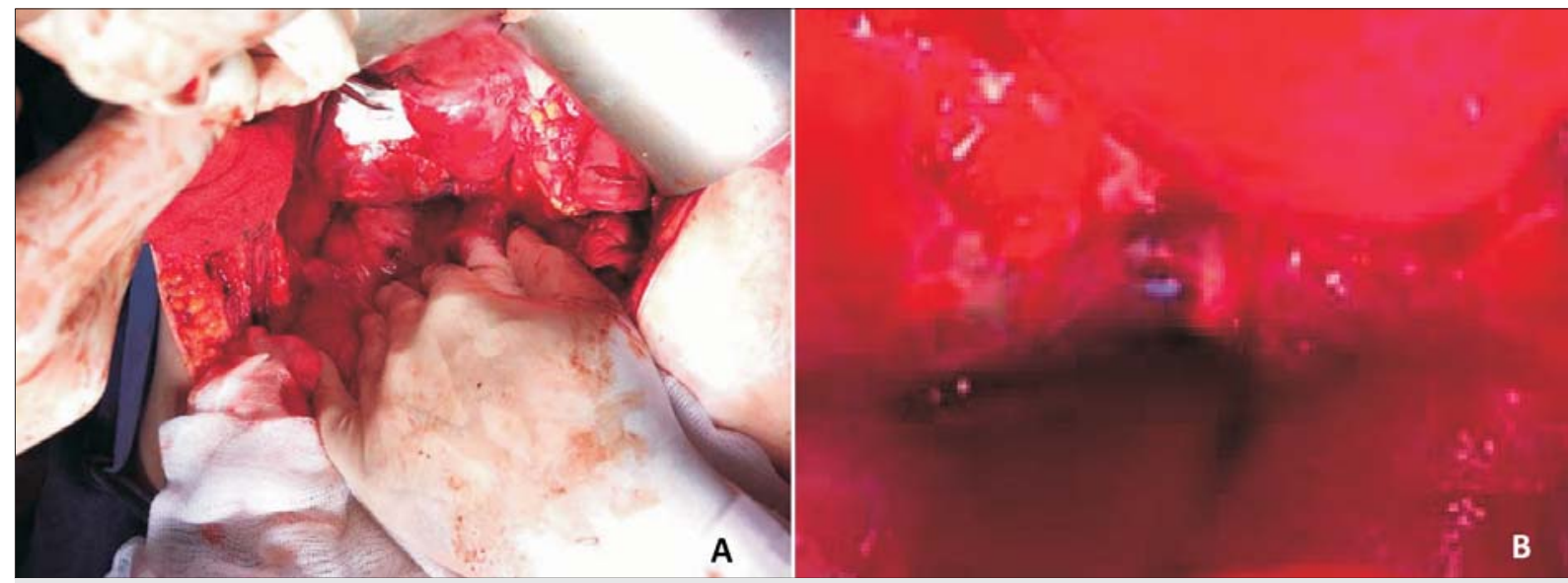

Figura 4. Pedículo portal en la segunda cirugía. A) Abordaje del pedículo portal derecho en la segunda cirugía. B) Evidencia del hilo irreabsorbible de la primera cirugía con el que se realizó la ligadura portal derecha. 

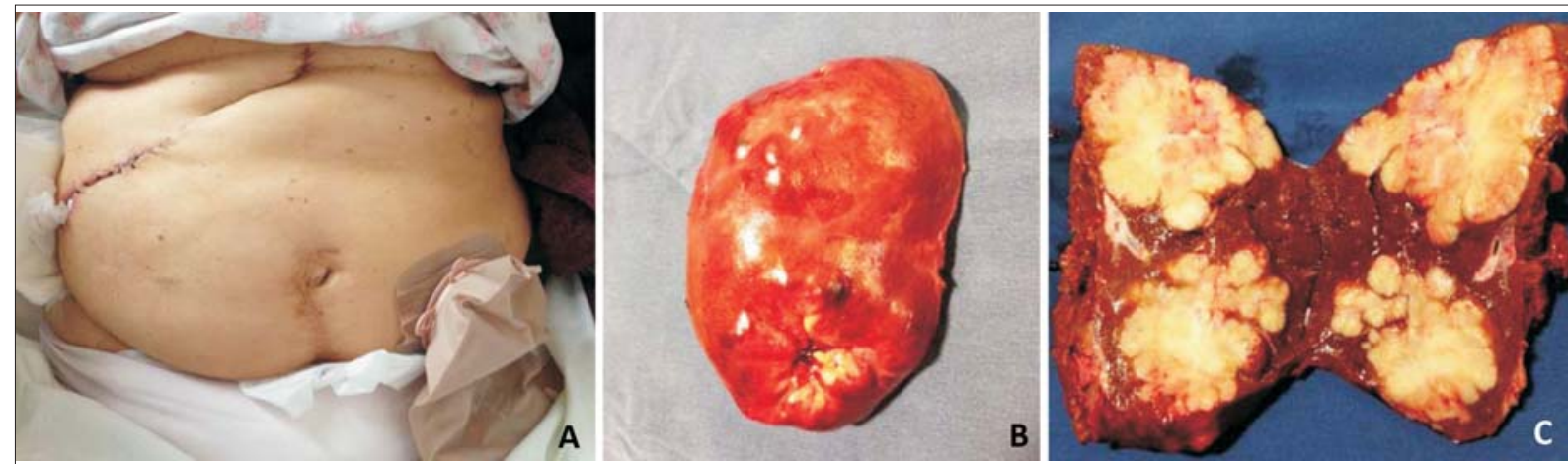

Figura 5. Posoperatorio luego de la segunda cirugía. A) Situación posoperatoria luego de la segunda cirugía. B) Pieza operatoria luego de la segunda cirugía. C) Apertura de la pieza visualizándose la ocupación casi total del hígado derecho por las metástasis.

\section{Discusión}

El abordaje de los pacientes con metástasis hepáticas colorrectales admite varias opciones terapéuticas, siendo el objetivo central lograr una resección completa del tumor con márgenes microscópicamente sanos. El concepto de la resecabilidad ha cambiado. Hoy día, todo tumor que puede ser resecado en su totalidad, dejando un adecuado hígado remanente, tiene indicación de cirugía $^{(8)}$. El porcentaje de hígado remanente necesario para que no se desarrolle una falla hepática posoperatoria es de $25 \%$ en hígados sanos, $30 \%$ en hígados con colestasis, hepatitis o quimioterapia y de $40 \%$ para hígados cirróticos. Esta reserva hepática funcional adecuada es un requisito indispensable para lograr un tratamiento exitoso y debe considerarse en la estrategia quirúrgica ${ }^{(9-11)}$.

La capacidad de regeneración del tejido hepático, así como la manipulación del flujo portal en busca de lograr una hipertrofia de parénquima hepático sano, ha permitido desarrollar lo que se conoce como cirugía hepática en dos tiempos (HDT). Si bien este concepto ha variado a lo largo de los años, en la actualidad existen dos tipos de cirugía en dos tiempos: la hepatectomía en dos tiempos con ligadura o embolización portal y la hepatectomía en dos tiempos que asocia una partición hepática in situ también conocida como ALPPS (Associating Liver Partition and Portal vein ligation for Staged hepatectomy). Esta última busca una hipertrofia acelerada del hígado funcional remanente en un tiempo más corto ${ }^{(12)}$. Sin embargo, presenta una mayor morbilidad y mortalidad que la hepatectomía en dos tiempos con ligadura portal clásica.

En nuestro caso, el cálculo preoperatorio del hígado funcional remanente era menor a $30 \%$, por lo que la metastasectomía del segmento 3 y la hepatectomía derecha en el mismo acto no era posible. Se requirió un procedimiento en dos tiempos. Dada la menor morbimortalidad de la HDT con ligadura portal, se optó por ella.
Durante el primer tiempo operatorio se busca limpiar el hígado remanente. Un punto de discusión, en este caso, estaba en hacer la metastasectomía quirúrgica o utilizar un método ablativo no resectivo, como la radiofrecuencia. El tamaño de la lesión mayor a $3 \mathrm{~cm}$, así como la relación contigua con el receso de Rex, hicieron que la mejor opción fuese la metastasectomía quirúrgica. En la estrategia propuesta por el equipo de R. Adam, en su trabajo pionero de 2004, se plantea que el abordaje de las metástasis hepáticas multinodulares bilobares depende de las características de las metástasis del hígado remanente ${ }^{(13)}$. Cuando estas son menores o iguales a 3 en número y menores a $30 \mathrm{~mm}$ de diámetro, lo recomendado es la hepatectomía del hígado con mayor carga tumoral y radiofrecuencia en las lesiones del hígado remanente. Sin embargo, cuando las lesiones del hígado remanente presentan un mayor tamaño o exceden en número a tres se aconseja la hepatectomía en dos tiempos, siendo esta situación la que presentó nuestra paciente (figura 6).

Este ejemplo clínico sirve para mostrar que la cirugía hepática ha experimentado un crecimiento exponencial en esta última década, presionando las fronteras de lo resecable. La utilización de fármacos oncológicos más efectivos, la mejoría en las imágenes preoperatorias, un mejor manejo posoperatorio y anestésico, así como cambios radicales en la técnica y táctica quirúrgica, ha llevado al tratamiento exitoso de pacientes con alta carga tumoral y un mal pronóstico, mejorando considerablemente su sobrevida ${ }^{(14,15)}$.

\section{Conclusión}

La hepatectomía en dos tiempos con oclusión de la vena porta es una estrategia quirúrgica aplicable en nuestro medio. Debe ser considerada en los pacientes con un buen performance status y una carga tumoral alta en los 


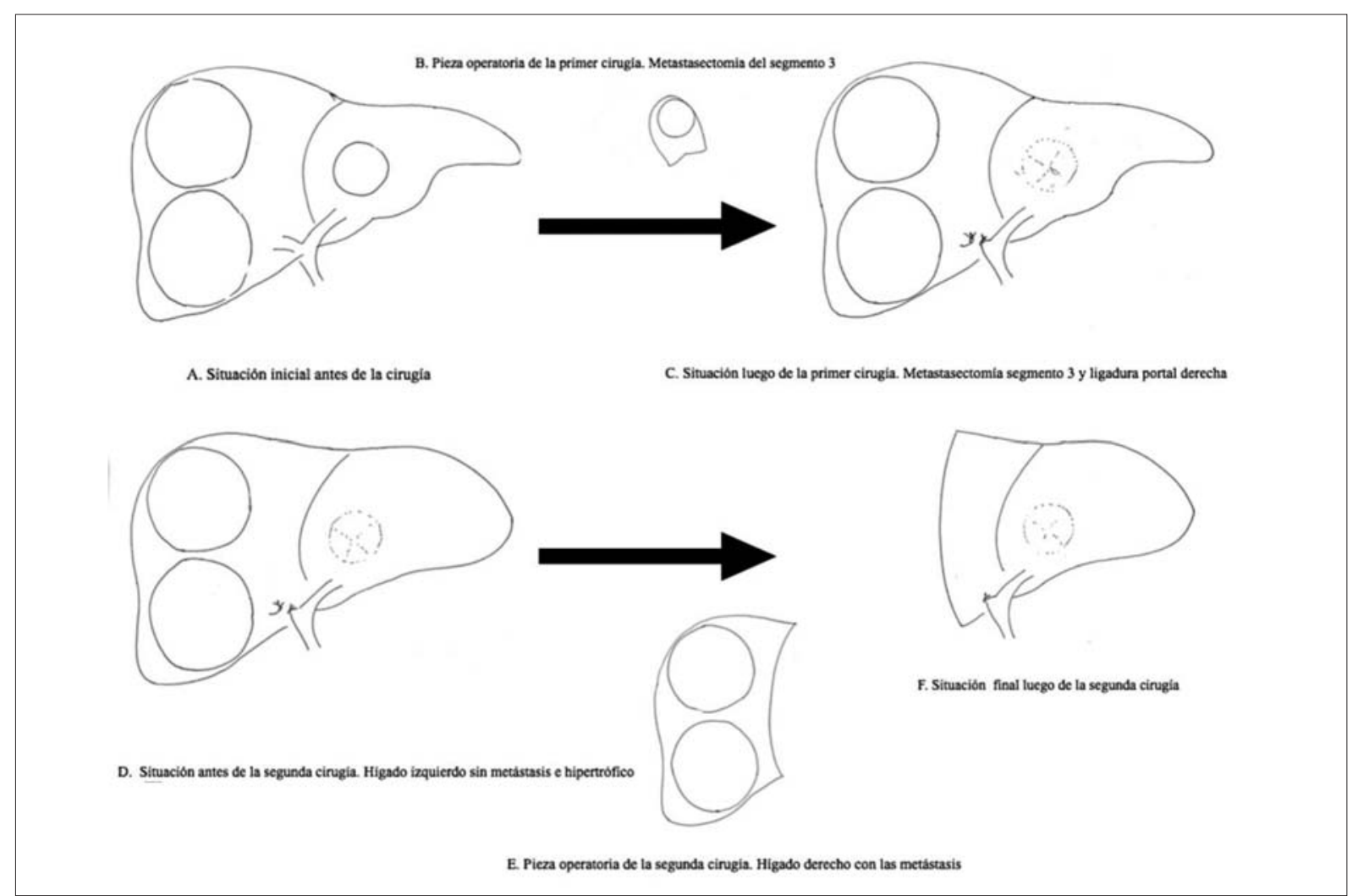

Figura 6. Pasos de la hepatectomía en dos tiempos con oclusión del flujo portal derecho realizados en esta paciente. A) Situación inicial. B) Pieza operatoria de la primera cirugía (metastasectomía del segmento 3). C) Primera cirugía. Metastasectomía del segmento 3 y ligadura portal derecha. D) Hipertrofia del hígado izquierdo, lográndose un volumen hepático residual mayor a 30\%. E) Pieza operatoria luego de la segunda cirugía. Hígado derecho con las dos metástasis. F) Segunda cirugía. Hepatectomía derecha.

que la resección, en un solo tiempo, llevaría a una insuficiencia hepática posoperatoria.

\section{Agradecimientos}

Al ex Residente de Cirugía, Dr. Fabián González, quien brindó asistencia a la paciente durante la internación y en policlínica.

Al Prof. Adjunto de la Clínica Quirúrgica "B”, Dr. Nicolás Muniz, así como a los asistentes Dres. Noelia Brito y Sebastián Fraga, quienes apoyaron y respaldaron la concreción de este caso clínico.

\section{Abstract}

Treatment of colorectal hepatic metastases has gradually changed in the last 20 years. Nowadays, surgical treatment remains one of the major curative treatment options available to patients. Survival in patients who may undergo a surgical resection of the tumor may add up to $40 \%$ in 5 years' time. These promising oncologic results have led surgical teams to make their best to achieve extreme liver resections which used to be contraindicated in the past. Thus, within this line of work, the liver remodeling surgery developed, which surgery consists in altering the portal flow aiming for a liver hypertrophy in the healthy liver and a liver atrophy of the sick liver. The following study aims to present one of the most frequent remodeling surgeries in the world: the two staged hepatectomy with portal flow occlusion. Indication, surgery opportunities, oncologic benefit and viability in our country are also discussed in the study.

\section{Resumo}

As opções terapêuticas para o tratamento das metástases hepáticas de origem colorretal mudaram muito nos últimos 20 anos. Atualmente a cirurgia é um pilar fundamental no tratamento dos pacientes portadores desta patologia. A sobrevida dos pacientes que podem ser submetidos a uma ressecção completa do tumor pode chegar a $40 \%$ em 5 anos. Estes resultados oncológicos tão estimulantes fazem com que as equipes cirúrgicas esforcem-se por conseguir ressecções hepáticas extre- 
mas que estavam contraindicadas anteriormente. Desenvolveu-se então, a cirurgia de remodelação hepática que consiste em alterar o fluxo portal buscando uma hipertrofia do fígado sadio e a atrofia do patológico. Descreve-se um caso que pretende mostrar uma das duas cirurgias de remodelação mais utilizadas no mundo - a hepatectomia em dois tempos com oclusão do fluxo portal. Discute-se a indicação, a oportunidade da cirurgia, o beneficio oncológico e a viabilidade da realização da mesma no nosso meio.

\section{Bibliografía}

1. Renehan AG, Egger M, Saunders MP, O'Dwyer ST. Impact on survival of intensive follow up after curative resection for colorectal cancer: systematic review and meta-analysis of randomised trials. BMJ 2002; 324:813.

2. Simmonds PC, Primrose JN, Colquitt JL, Garden OJ, Poston GJ, Rees M. Surgical resection of hepatic metastases from colorectal cancer: a systematic review of published studies. Br J Cancer 2006; 94:982-99.

3. Pawlik TM, Schulick RD, Choti MA. Expanding criteria for resectability of colorectal liver metastases. Oncologist 2008; 13:51-64. doi: 10.1634/theoncologist.2007-0142.

4. Hadden WJ, de Reuver PR, Brown K, Mittal A, Samra JS, Hugh TJ. Resection of colorectal liver metastases and extra-hepatic disease: a systematic review and proportional meta-analysis of survival outcomes. HPB (Oxford) 2016; 18:209-20. doi: 10.1016/j.hpb.2015.12.004.

5. Abdalla EK, Vauthey JN, Ellis LM, Ellis V, Pollock R, Broglio KR, Hess K, Curley SA. Recurrence and outcomes following hepatic resection, radiofrequency ablation, and combined resection/ablation for colorectal liver metastases. Ann Surg 2004; 239:818-5.

6. Jaeck D, Oussoultzoglou E, Rosso E, Greget M, Weber JC, Bachellier P. A two-stage hepatectomy procedure combined with portal vein embolization to achieve curative resection for initially unresectable multiple and bilobar colorectal liver metastases. Ann Surg 2004; 240(6):1037-49.
7. Kianmanesh R, Farges O, Abdalla EK, Sauvanet A, Ruszniewski P, Belghiti J. Right portal vein ligation: a new planned two-step all-surgical approach for com- plete resection of primary gastrointestinal tumors with multiple bilateral liver metastases. J Am Coll Surg 2003; 197(1):164-70.

8. Pawlik TM, Scoggins CR, Zorzi D, Abdalla EK, Andres A, Eng C, et al. Effect of surgical margin status on survival and site of recurrence after hepatic resection for colorectal metastases. Ann Surg 2005; 241(5):715-22.

9. Dahm F, Georgiev P, Clavien PA. Small-for-size syn- drome after partial liver transplantation: definition, mechanisms of disease and clinical implications. Am J Transplant 2005; 5(11):2605-10.

10. Balzan S, Belghiti J, Farges O, Ogata S, Sauvanet A, Delefosse $\mathrm{D}$, et al. The "50-50 criteria" on postopera- tive day 5 : an accurate predictor of liver failure and death after hepatectomy. Ann Surg 2005; 242(6):824-8.

11. Rahbari NN, Garden OJ, Padbury R, Brooke-Smith M, Crawford M, Adam R, et al. Posthepatectomy liver failure: a definition and grading by the International Study Group of Liver Surgery (ISGLS). Surgery 2011; 149(5):713-24.

12. de Santibañes E, Clavien PA. Playing Play-Doh to prevent postoperative liver failure: the "ALPPS" approach. Ann Surg 2012; 255(3):415-7.

13. Adam R, Wicherts DA, de Haas RJ, Ciacio O, Levi F, Paule B, et al. Patients with initially unresectable colorectal liver metastases: is there a possibility of cure? J Clin Oncol 2009; 27(11):1829-35.

14. Bipat S, van Leeuwen MS, Comans EF, Pijl ME, Bossuyt PM, Zwinderman AH, et al. Colorectal liver metastases: CT, MR imaging, and PET for diagno-sis-meta-analysis. Radiology 2005; 237(1):123-31.

15. Breitenstein S, Apestegui C, Petrowsky H, Clavien PA. "State of the art" in liver resection and living donor liver transplantation: a worldwide survey of 100 liver centers. World J Surg 2009; 33(4):797-803. 